\title{
Effects of cardiac sympathetic innervation on regional wall motion abnormality in patients with long QT syndrome
}

\author{
H Yamanari, K Nakayama, H Morita, K Miyazi, K Fukushima, H Matsubara, T Emori,
} T Ohe

\begin{abstract}
Aim-To assess the spatial relation between regional cardiac sympathetic innervation and regional ventricular repolarisation indicated by ventricular wall motion abnormality in patients with congenital long QT syndrome.

Design-Regional percentage uptake and washout rate of ${ }^{123}$ I metaiodobenzylguanidine (MIBG) were measured to assess cardiac sympathetic innervation in septum, anterior wall, lateral wall, and posterior wall. Left ventricular short axis images on echocardiography were digitised to reconstruct digitised $\mathrm{M}$ mode echocardiograms, from which left ventricular wall thickness curves were obtained. The wall thickening time (ThT) was defined as the period in which the instantaneous wall thickness exceeded $90 \%$ of the maximum wall thickness. The ThT was measured from the ventricular wall thickness curve at the same segments where regional percentage uptake and washout rate of ${ }^{123}$ I MIBG were measured.

Patients-Seven patients with long QT syndrome.

Results-The regional washout rate (mean (SD)) of ${ }^{123} \mathrm{I}$ MIBG in patients with long QT syndrome was greater in the segments with decreased percentage uptake of ${ }^{123}$ I MIBG than in those without $(17.4(10.6) \% v 9.7(16.5) \%, \mathrm{p}<0.03)$. ThT in segments both with and without decreased percentage uptake of ${ }^{123}$ I MIBG was longer than in control subjects $(p<0.0001)$. ThT was longer in the segments with decreased percentage uptake of ${ }^{123}$ I MIBG than in those without (199 (70) ms v 150 (66) ms, $\mathrm{p}=0.0018$ ).

Conclusions-Activation of regional cardiac sympathetic terminals is likely to participate in additional regional prolongation of ventricular repolarisation in patients with long QT syndrome. (Heart 2000;83:295-300)
\end{abstract}

Keywords: long QT syndrome; sympathetic innervation; regional wall motion

Prolonged ventricular repolarisation and lethal ventricular tachyarrhythmias are characteristic of patients with congenital long QT syndrome. ${ }^{1-3}$ Previous studies ${ }^{3}{ }^{4}$ have suggested that increased heterogeneity of regional ventricular repolarisation is involved in the occurrence of polymorphic ventricular tachycardia. This ventricular repolarisation has recently been assessed not only by electrophysiological indices such as QT interval and monophasic action potential duration but also by the mechanical index of ventricular wall motion. ${ }^{5}$ Although the QT interval in standard 12 lead ECG is a conventional electrophysiological index of ventricular repolarisation, it is not known whether the QT interval of each lead in the ECG indicates ventricular repolarisation in any site of the ventricle. However, non-invasive assessment of regional ventricular wall motion using echocardiography allows quantification of the spatial variation of ventricular repolarisation.

Mutations in cardiac potassium channel genes and in the sodium channel gene have been confirmed in patients with long QT syndrome. ${ }^{67}$ However, mutations in these genes cannot alone explain the mechanism of regional heterogeneity of ventricular repolarisation. Unbalanced sympathetic innervation has been suggested as one of the substrates, and a sudden increase in sympathetic tone as the trigger for lethal ventricular tachyarrhythmias. ${ }^{12}$ Based on the assumed pathogenesis of the long QT syndrome, treatment with $\beta$ blockers or left heart sympathetic denervation is highly successful in preventing sudden cardiac death. ${ }^{2}$ Thus it is important to evaluate the relation between regional ventricular repolarisation and ventricular sympathetic innervation.

For non-invasive detection of impaired presynaptic cardiac sympathetic nerve endings, ${ }^{123} \mathrm{I}$ metaiodobenzylganidine (MIBG) scintigraphy has been used in patients with long QT syndrome as well as in those with coronary heart disease and cardiomyopathy. ${ }^{8}$ It is possible to assess the spatial variation in regional sympathetic innervation in patients with long QT syndrome using ${ }^{123} \mathrm{I}$ MIBG single photon emission computed tomography (SPECT). Our aim in this study was to assess the spatial relation between regional cardiac sympathetic innervation using ${ }^{123}$ I MIBG SPECT and regional ventricular repolarisation assessed by ventricular wall motion abnormality using a digitised wall thickness curve in patients with long QT syndrome.

\section{Methods}

SUBJECTS

Seven patients (five male and two female, mean (SD) age 34 (22) years, range 13 to 66 years) were studied with their informed consent according to the Declaration of Helsinki. Long QT syndrome was defined according to the 
Table 1 Clinical characteristics of the study patients

\begin{tabular}{lllllllll}
\hline No & $\begin{array}{l}\text { Age } \\
\text { (years) }\end{array}$ & Sex & Diagnosis & $\begin{array}{l}\text { Syncope } \\
\text { TdP }\end{array}$ & $\begin{array}{l}\text { Sinus cycle } \\
\text { length (ms) }\end{array}$ & $\begin{array}{l}\text { QT } \\
(m s)\end{array}$ & $\begin{array}{l}\text { QTc } \\
\left(m s^{1 / 2}\right)\end{array}$ & $\begin{array}{l}\text { Decreased segments of } \\
\text { 123 IMIBG\% uptake }\end{array}$ \\
\hline 1 & 17 & M & RW & + & 1090 & 560 & 540 & IVS \\
2 & 13 & M & RW & - & 1020 & 500 & 500 & IVS \\
3 & 23 & F & Idp & + & 1150 & 620 & 510 & IVS \\
4 & 48 & F & RW & + & 1050 & 600 & 570 & AW \\
5 & 66 & M & Idp & - & 1360 & 580 & 500 & LW, PW \\
6 & 17 & M & Idp & + & 1380 & 600 & 510 & IVS \\
7 & 57 & M & RW & + & 1300 & 560 & 490 & IVS, LW \\
\hline
\end{tabular}

AW, anterior wall; Idp, idiopathic long QT syndrome; IVS, interventricular septum; LW, lateral wall; MIBG, metaiodo benzylguanidine; PW, posterior wall; QTc, corrected QT interval; RW, Romano-Ward syndrome; TdP, torsade de pointes.

A

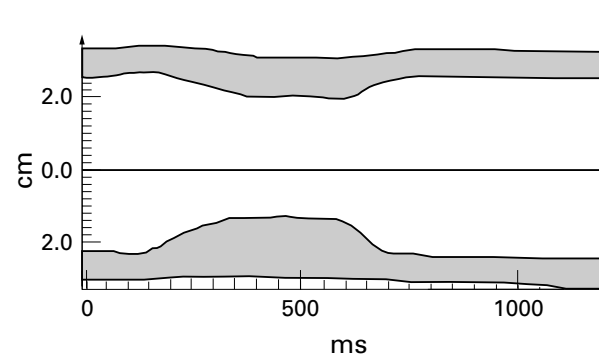

B

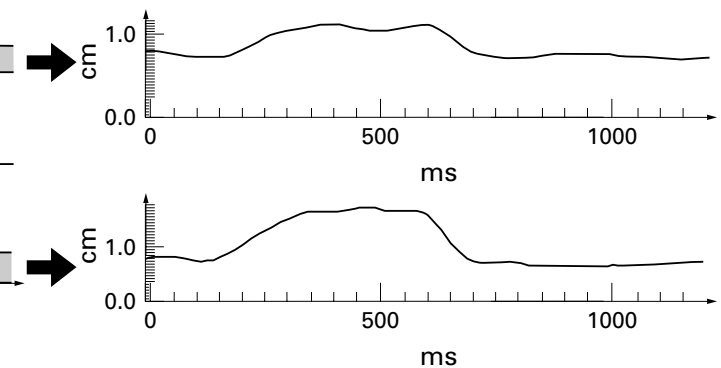

Figure 1 Panel $A$ shows digitised $M$ mode echocardiograms of mid-septum and lateral wall reconstructed from left ventricular short axis images in a patient with long $Q T$ syndrome. Panel B shows wall thickness curves obtained from tracing the digitised M mode echocardiograms at each measurement site. (Modified from reference 10.)

diagnostic criteria of Schwartz et al. ${ }^{9}$ Four patients had familial long QT syndrome (Romano-Ward syndrome) and three had sporadic long QT syndrome. All patients with long QT syndrome had a QTc interval longer than $480 \mathrm{~ms}^{1 / 2}$ (range $490 \mathrm{~ms}^{1 / 2}$ to $570 \mathrm{~ms}^{1 / 2}$ ), which was unrelated to electrolyte abnormality or any other cause of QT prolongation. Five patients had a history of syncope or torsade de pointes (table 1). Three had a notched $\mathrm{T}$ wave in three leads. $T$ wave alternans was not present in any patient. None of the patients had a history of angina pectoris or myocardial infarction. Pathological ST segment depression was not observed during exercise testing in any of the patients. Diabetes mellitus and other metabolic diseases were excluded in all patients. None of the patients had been taking any antiarrhythmic drugs or $\beta$ blockers, or any drugs interfering with the biodistribution of ${ }^{123}$ I MIBG, such as reserpine, antidepressants, guanethidine, and calcium channel blockers. ${ }^{10}$

Nine control subjects without obvious cardiac or lung disease were also studied (six male, three female, age 23 (7) years). They had QTc intervals shorter than $430 \mathrm{~ms}^{1 / 2}$ (range 350 $\mathrm{ms}^{1 / 2}$ to $430 \mathrm{~ms}^{1 / 2}$, mean $390(20) \mathrm{ms}^{1 / 2}$ ).

\section{ECHOCARDIOGRAPHY}

Echocardiography was performed using a commercially available device (Sonos 1500, Hewlett-Packard, Andover, Massachusetts, USA) equipped with $3.5 \mathrm{MHz}$ and $2.5 \mathrm{MHz}$ transducers. Standard cross sectional, M mode, and Doppler echocardiographic studies were performed in patients with long QT syndrome and controls. The echocardiography was recorded on videotape together with an ECG in lead II.
IMAGE ACQUISITION AND THE WALL THICKNESS CURVE

Left ventricular short axis images at the basal and middle levels were recorded on half inch videotape. The wall thickness curve and its digitised $M$ mode were obtained from the videotape, as previously described. ${ }^{11}$ The recorded short axis images were preserved as consecutive digitised B mode images every 33 $\mathrm{ms}$ in an offline image analysing computer (Mipron, Kontron Elektronik, Munich, Germany), and we reconstructed the digitised $M$ mode echocardiograms from these images. We obtained regional left ventricular wall thickness curves from these digitised $M$ mode echocardiograms (fig 1). The wall thickening time (ThT) on the wall thickness curve was then measured. ThT was defined as the period during which wall thickness exceeded $90 \%$ of maximum (fig 2). It was measured at three sites in both the septum (anterior septum, midseptum, and posterior septum) and the free wall (anterior wall, lateral wall, and posterior wall) of the left ventricle, so a total of 12 sites was used for ThT measurements. The value of

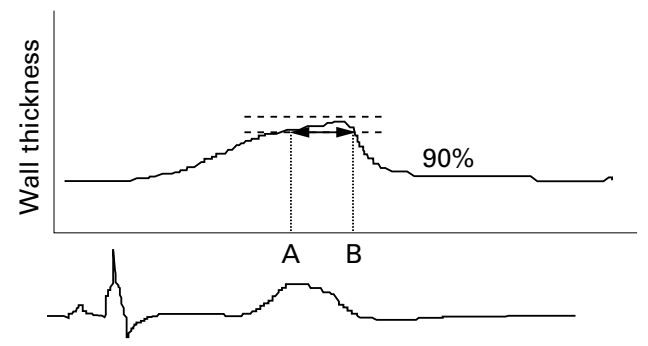

Figure 2 Schema shows wall thickness curve of the left ventricular wall at one measurement site together with the ECG. Segment AB represents wall thickening time (ThT), defined as the period in which the late systole exceeded $90 \%$ of the maximum wall thickness. (Modified from reference 10.) 
ThT in the septum was the averaged value of all three sites. Thus we evaluated ThT in eight segments (septum, anterior wall, lateral wall, and posterior wall at the basal and middle levels) in the present study. The reproducibility of the measurement of ThT was assessed twice by the same observer to assess interobserver reproducibility, as well as by two observers for intraobserver reproducibility. The mean difference in interobserver reproducibility was 12 (26) $\mathrm{ms}$, and for the intraobserver reproducibility, 2 (14) ms.

SCINTIGRAPHIC METHODS OF ${ }^{123}$ I MIBG

SPECT was performed with a single head gamma camera (GCA-901A/HG, Toshiba, Tokyo, Japan) equipped with a low energy general purpose collimator centered on the 159 $\mathrm{KeV}$ photo peak with a $20 \%$ window for ${ }^{123} \mathrm{I}$. At rest $111 \mathrm{MBq}$ of ${ }^{123} \mathrm{I}$ MIBG were injected intravenously. Thirty six projections were obtained with a $128 \times 128$ matrix for 30 seconds each in a $180^{\circ}$ arc at 30 minutes after intravenous injection (early image) and after four hours (delayed image). The short axis images were reconstructed by the raw projection data. For quantitative analysis in the basal and middle levels of the left ventricle, a circumferential profile analysis of cardiac uptake of ${ }^{123} \mathrm{I}$ MIBG was performed in three summed short axis images at each level. The septum, inferior wall, lateral wall, and anterior wall-which corresponded to the measured sites in echocardiography - were defined as the segments numbered 8-6, 4, 3, and 2, respectively (fig 3). Regional percentage uptake of ${ }^{123} I$ MIBG in each segment was calculated by the ratio of segmental uptake counts of ${ }^{123}$ I MIBG to maximum uptake counts in the delayed image. ${ }^{12}$ A "decreased segment" on regional percentage uptake of ${ }^{123}$ I MIBG was defined as a segment with a value 2 SD below the mean for normal volunteers.

The regional washout rate of ${ }^{123} \mathrm{I}$ MIBG in each segment was calculated by the following formula: (regional uptake counts of ${ }^{123}$ I MIBG in the early image - regional uptake counts of ${ }^{123} \mathrm{I}$ MIBG in the delayed image) $\div$ regional uptake counts of ${ }^{123}$ I MIBG in the early image.

Normal values for regional percentage uptake and washout rate of ${ }^{123}$ I MIBG were obtained from 18 normal Japanese volunteers (nine men and nine women, mean age 48 (15) years) at our hospital by ${ }^{123}$ I MIBG scintigraphy. The volunteers were physically normal and had normal ECGs and echocardiograms.

\section{STATISTICAL ANALYSIS}

Data are expressed as mean (SD). Statistical significance was accepted when $\mathrm{p}<0.05$. Comparison between groups was performed by
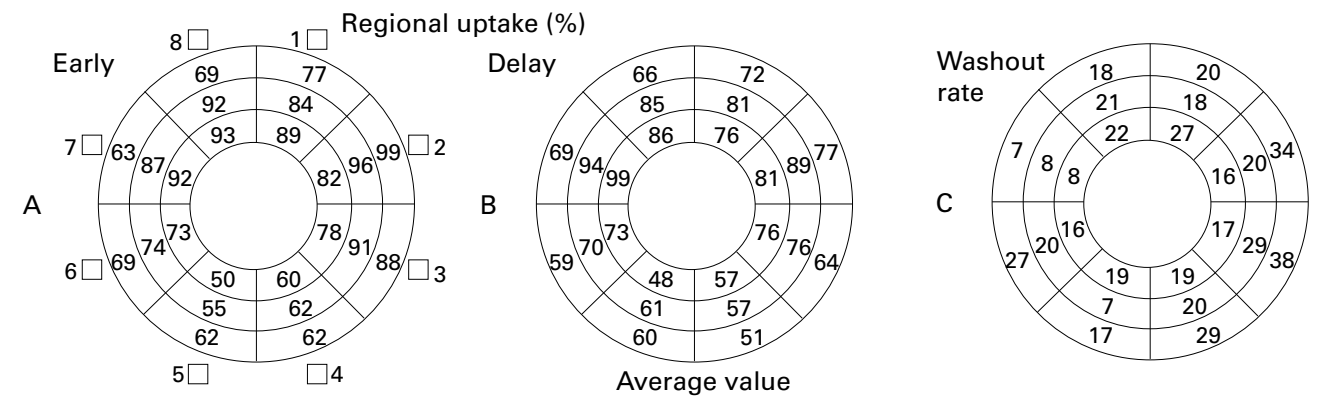

Figure 3 Panels $A, B$, and $C$ show bull's eye schema of quantitative ${ }^{123} I M I B G$ scintigraphy in a patient with long $Q T$ syndrome. Sectors of the left ventricle are displayed from the apical side (centre of the schema) to the basal side (periphery of the schema). The septum, inferior wall, lateral wall, and anterior wall are defined as the segments numbered 8-6, 4, 3, and 2 , respectively. Panels $A$ and $B$ show regional percentage uptake of ${ }^{123} I M I B G$ at an early image and a delayed image, respectively. Panel $C$ shows regional washout rate calculated from regional uptake counts of ${ }^{123} I M I B G$. MIBG, metaiodobenzylguanidine.
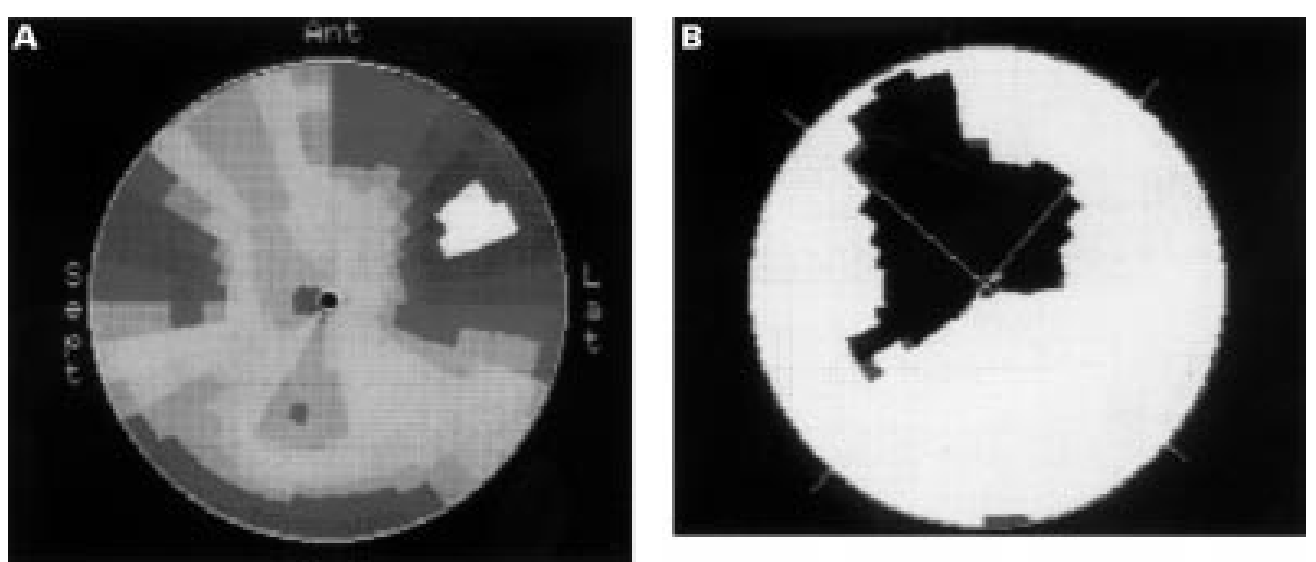

Figure 417 year old man with idiopathic long $Q T$ syndrome. Bull's eye polar map of ${ }^{123} I$ MIBG delayed image shows reduced uptake of ${ }^{123} I M I B G$ in the septum, inferior wall, and apex $(A)$. However, the segments showing decreased regional percentage uptake of ${ }^{123} I M I B G$, that is segments with a value $2 S D$ below the mean for normal volunteers were noted in the basal and mid septum, and in the apex (B). The black area in panel $B$ indicates the segment with decreased regional percentage uptake of ${ }^{123} I$ MIBG. MIBG, metaiodobenzylguanidine. 


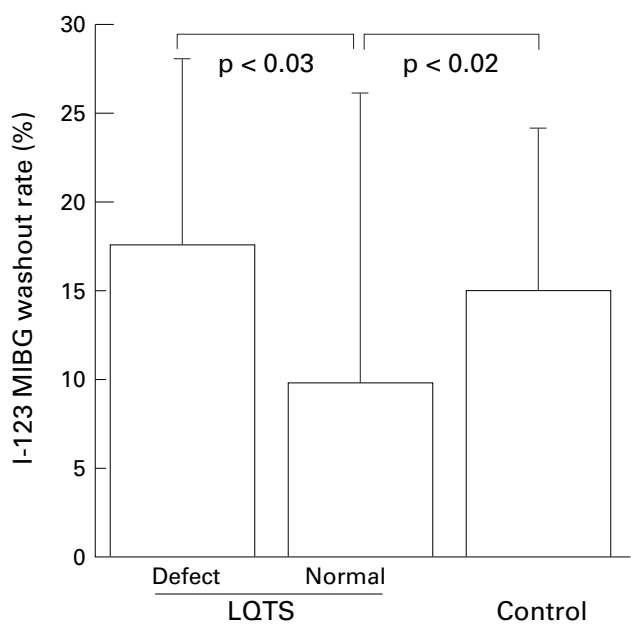

Figure 5 Regional washout rate of ${ }^{123}$ I MIBG in patients with long $Q T$ syndrome and control subjects. The regional washout rate of ${ }^{123} I M I B G$ in patients with long $Q T$ syndrome was greater in the segments with decreased percentage uptake of ${ }^{123} I$ MIBG than in those without. Defect, patients with decreased percentage uptake of ${ }^{123} I$ $M I B G ; L Q T S$, long $Q T$ syndrome; $M I B G$,

metaiodobenzylguanidine; Normal, patients without decreased percentage uptake of ${ }^{123} I M I B G$.

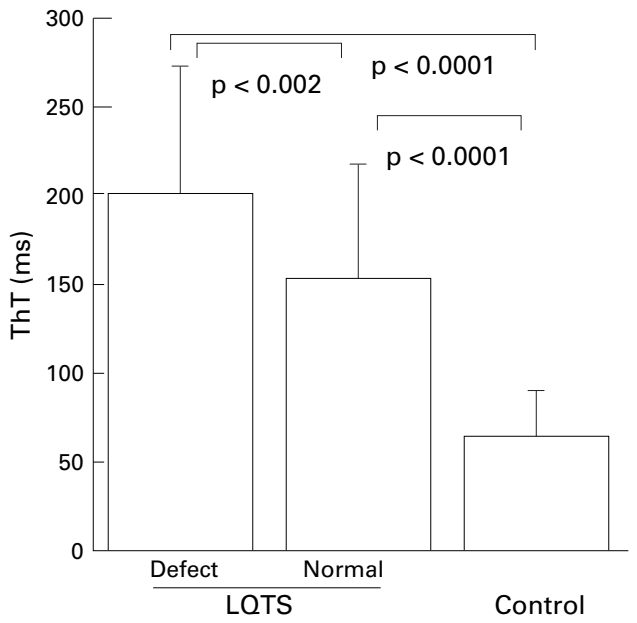

Figure 6 Graph showing comparison between wall thickening time (ThT) and regional percentage uptake of ${ }^{123} I M I B G$. ThT in patients with long $Q T$ syndrome was significantly longer in the segments with decreased percentage uptake of ${ }^{123} I$ MIBG than in this without. Defect, patients with decreased percentage uptake of ${ }^{123} I$ $M I B G ; L Q T S$, long $Q T$ syndrome; $M I B G$, metaiodobenzylguanidine; Normal, patients without decreased percentage uptake of ${ }^{123} I M I B G$.

analysis of variance (ANOVA) and Bonferroni's test.

\section{Results}

PERCENTAGE UPTAKE AND WASHOUT RATE OF ${ }^{123}$ I MIBG

Segments with decreased percentage uptake of ${ }^{123}$ I MIBG were observed in all seven patients with long QT syndrome. Of these, five had segments with decreased regional uptake located in the septum, two had segments in the anterior wall, two in the lateral wall, and one in the inferior wall (fig 4). The regional washout rate of ${ }^{123} \mathrm{I}$ MIBG in patients with long QT syndrome was greater in the segments with decreased percentage uptake of ${ }^{123}$ I MIBG than in those without $(17.4 \quad(10.6) \% \quad v \quad 9.7 \quad(16.5) \%$, $\mathrm{p}<0.03)$. Regional washout rate of ${ }^{123}$ I MIBG was less in segments without decreased percentage uptake of ${ }^{123} \mathrm{I}$ MIBG in patients with the long QT syndrome than the control subjects $(9.7(16.5) \%$ v $14.9(9.3) \%, \mathrm{p}<0.02)$ (fig 5).

COMPARISON BETWEEN ThT AND PERCENTAGE UPTAKE OF ${ }^{123}$ I MIBG

ThT values in segments both with and without decreased percentage uptake of ${ }^{123}$ I MIBG were longer than in control subjects $(p<0.0001)$. ThT was longer in the segments with decreased percentage uptake of ${ }^{123}$ I MIBG than in those without $(199$ (70) $\mathrm{ms} v 150$ (66) $\mathrm{ms}$, $\mathrm{p}<0.002$ ) (fig 6).

\section{Discussion}

Our major findings in this study of patients with long QT syndrome were first, there was a decreased regional percentage uptake of ${ }^{123} \mathrm{I}$ MIBG; second, the washout rate of ${ }^{123}$ I MIBG was greater in segments with decreased percentage uptake of ${ }^{123}$ I MIBG than those with normal uptake; and third, ThT was significantly longer in the segments with the decreased percentage uptake of ${ }^{123}$ I MIBG than in those without.

There have been several studies on cardiac sympathetic innervation, ${ }^{813-15}$ assessed by ${ }^{123} \mathrm{I}$ MIBG SPECT or positron emission tomography using C-11 hydroxyephedrine (HED), in patients with the long QT syndrome. Their conclusions are far from consistent. Eisen $e t a l,{ }^{8}$ Göhl et $a l l^{13}$ and Müller et $a l^{14}$ reported the presence of a significant decrease in ${ }^{123}$ I MIBG uptake in affected individuals. In contrast, Calkins et al found no difference in HED uptake between long QT syndrome patients and normal controls. ${ }^{15}$ Our results showed a significant decrease in regional percentage uptake of ${ }^{123} \mathrm{I}$ MIBG in all patients. The discrepancy between our results and those of previous studies may reflect the rather small numbers of patients and the wide spectrum of long QT syndrome.

Heterogeneous uptake of ${ }^{123} \mathrm{I}$ MIBG in the left ventricle has been observed in normal individuals. Previous studies in normal subjects indicated that regional uptake of ${ }^{123}$ I MIBG in the septum and inferior wall was less than in the other wall of the left ventricle. ${ }^{16}{ }^{17}$ In the present study, we defined a segment with decreased regional percentage uptake of ${ }^{123} \mathrm{I}$ MIBG as one with values that were 2 SD below the mean in normal volunteers. Hence we were able to evaluate segments showing decreased ${ }^{123}$ I MIBG uptake despite the problem of heterogeneous ${ }^{123}$ I MIBG uptake in the left ventricle. Decreased ${ }^{123} \mathrm{I}$ MIBG uptake is observed when there are defects in myocardial sympathetic nerve terminals or an increase in the level of sympathetic activity. Sisson et al reported that both an increased washout rate of ${ }^{123}$ I MIBG and a decreased uptake of ${ }^{123}$ I MIBG in the heart indicated activation of cardiac sympathetic neurones. ${ }^{18}$ In the present study, the segments with decreased uptake of ${ }^{123} \mathrm{I}$ MIBG had an increased washout rate of ${ }^{123} \mathrm{I}$ MIBG compared with those without decreased 
uptake. This suggests that there is relative activation of regional cardiac sympathetic terminals in patients with the long QT syndrome.

Evidence of a mechanical disturbance as well as an electrophysiological abnormality was observed in patients with the long QT syndrome. Nakayama et al reported that regional wall thickening time dispersion was significantly correlated with QT dispersion. ${ }^{11}$ This suggests that dispersion of ventricular electrical repolarisation contributes to the dispersion of mechanical ventricular wall motion abnormality. The assessment of regional wall thickening time allows quantification of the spatial variation of ventricular repolarisation. Wall thickening time in the present study was significantly prolonged in the long QT syndrome, regardless of the degree of uptake of ${ }^{123}$ I MIBG, compared with the control subjects. Recently, it was found that mutation of the cardiac potassium channel gene or the sodium channel gene causes the long QT syndrome. ${ }^{19}{ }^{20}$ Cardiac repolarisation represents a delicate balance between inward and outward currents during the plateau phase of the cardiac action potential. Either persistent inward current (for example, through sodium channels) or reduced outward current (through potassium channels) can cause prolongation of the action potential. When the mutant HERG gene identified as the cause of the long QT syndrome was expressed in Xenopus oocytes, a reduction of outward current was recorded..$^{19}$ Mutation of SCN5A, the gene encoding the cardiac sodium channel $\alpha$ subunit, produces a small sustained inward current. ${ }^{20}$ This small inward current is most probably sufficient to disrupt the normal balance between inward and outward currents during the plateau phase. These findings indicate that mutation of the cardiac potassium channel gene or the sodium channel gene contributes to prolonged ventricular repolarisation in the long QT syndrome. However, wall thickening time was significantly longer in the segments with decreased uptake of ${ }^{123}$ I MIBG than in those without. Moreover, Nador et al found that a left ventricular wall motion abnormality similar to that observed in the long QT syndrome was provoked in dogs after right stellectomy. ${ }^{5}$ Thus heterogeneous activation of regional cardiac sympathetic terminals, in addition to mutation of ion channels, is likely to participate in the heterogeneity of ventricular repolarisation in patients with this syndrome. However, we cannot clearly explain the mechanism of ventricular wall motion abnormality associated with relative activation of cardiac sympathetic terminals. A previous study ${ }^{21}$ showed that ventricular wall motion abnormality in patients with the long QT syndrome was abolished by the injection of a calcium channel blocker. This suggests that the myocardium in which sympathetic terminals are activated may have an abnormal increase in intracellular calcium concentration before complete relaxation has occurred, and that prolonged myocardial contraction may result.

Wall thickening time was significantly longer in segments with decreased uptake of ${ }^{123} \mathrm{I}$
MIBG than in those without. Regional percentage uptake of ${ }^{123} \mathrm{I}$ MIBG only indicated relative regional uptake of ${ }^{123} \mathrm{I}$ MIBG, whereas wall thickening time indicated the absolute value of regional wall motion abnormality, not a relative value. Even if regional myocardial sympathetic innervation or activity was equal among the patients, the regional uptake of ${ }^{123} \mathrm{I}$ MIBG might differ. We therefore did not assess the correlation between regional percentage uptake of ${ }^{123} \mathrm{I}$ MIBG and the wall thickening time. At a future date, it will be necessary to show a direct correlation between regional myocardial sympathetic innervation and wall motion abnormality.

\section{LIMITATIONS}

There are several limitations to our study. The numbers of patients and control subjects were small. Genetic analysis for identification of disorders causing the long QT syndrome mutation was not performed. Thus our results may only reflect a part of the wide spectrum of long QT syndrome.

The segments evaluated for wall thickening time and percentage uptake of ${ }^{123}$ I MIBG were only in the basal and mid-left ventricular regions. Uptake counts of ${ }^{123} \mathrm{I}$ MIBG in the right ventricle were low, and true apical short axis images in echocardiography could not be recorded precisely, so we could not evaluate wall motion and ${ }^{123}$ I MIBG uptake in the whole of the left and right ventricles.

We recorded the images on videotape. Videotape imaging is limited to 30 frames a second, so we used a smoothing method by interpolation to obtain the smooth digitised $\mathrm{M}$ mode echocardiograms. Cross sectional echocardiography recorded on videotape is limited by time dependent analysis.

None of the patients had been taking any drugs that would interfere with the biodistribution of ${ }^{123}$ I MIBG. Further, ${ }^{123}$ I MIBG scintigraphy was performed while the patients were fully rested. Because cardiac sympathetic activation was not affected by any factors except intrinsic activity, we did not investigate the reproducibility of ${ }^{123}$ I MIBG scintigraphy.

\section{CONCLUSIONS}

We assessed the relation between regional cardiac sympathetic innervation using ${ }^{123} \mathrm{I}$ MIBG SPECT and regional ventricular repolarisation, indicated by ventricular wall motion abnormality, using digitised wall thickness curves in patients with the long QT syndrome. Decreased percentage uptake of ${ }^{123} \mathrm{I}$ MIBG and increased washout rate of ${ }^{123}$ I MIBG were observed regionally in locations where there was more prolonged wall thickening time. This suggests that activation of regional cardiac sympathetic terminals in addition to mutations of ion channels is likely to participate in regional prolongation of ventricular repolarisation in patients with the long QT syndrome. The conclusions of the current study are speculative and further larger studies are necessary to confirm them. 
1 Schwartz PJ, Periti M, Malliani A. The long Q-T syndrome. Am Heart f 1978;89:378-90.

Progress and questions. Am Heart f 1985;109:399-411.

3 Jackman WN, Friday KJ, Anderson JL, et al. The long QT syndromes: a critical review, new clinical observations and a unifying hypothesis. Prog Cardiovasc Dis 1988;31:115-72.

4 Surawicz B. Electrophysiologic substrate of torsade de pointes: dispersion of repolarization or early afterrepolarizations? 7 Am Coll Cardiol 1989;14:172-84

5 Nador F, Beria G, De Ferrari GM, et al. Unsuspected echocardiographic abnormality in the long QT syndrome. Circulation 1991;84:1530-42.

6 Curran ME, Splawski I, Timothy KW, et al. A molecular basis for cardiac arrhythmia: HERG mutations cause long QT syndrome. Cell 1995;80:795-803.

7 Wang Q, Shen J, Splawski I, et al. SCN5A mutations associated with an inherited cardiac arrhythmia, long QT syndrome. Cell 1995;80:805-11.

8 Eisen HJ, Nader RG, Eng-Ter S, et al. Noninvasive detection of regional abnormalities in myocardial adrenergic innervaof regional abnormalities in myocardial adrenergic innerva-
tion in patients with idiopathic long QT syndrome using tion in patients with idiopathic long QT syndrome using I-123-metaiodobenzylgua

9 Schwartz PJ, Locati EH, Napolitano C, et al. The long QT syndrome. In: Zipes DP, Jalife J, eds. Cardiac electrophysiology: from cell to bedside. Philadelphia: WB Saunders Co, 1995:788-811.

10 Solanki KK, Bomanji J, Moyes J, et al. A pharmacologica guide to medicine which interfere with the biodistribution of radiolabelled meta-iodobenzylguanidine (MIBG). Nucl Med Commun 1992;13:513-21.

11 Nakayama K, Yamanari H, Otsuka F, et al. Dispersion of regional wall motion abnormality in patients with long QT syndrome. Heart 1998;80:245-50.
12 Stirner H, Bull U, Kleinhans E. Three-dimensional ROI-based quantificationof stress/rest ${ }^{201} \mathrm{Tl}$ myocardial
SPECT: presentation of method. Nucl Med 1986;25:12833.

13 Göhl K, Feistel H, Weikl A, et al. Congenital myocardial sympathetic dysinnervation (CMSD). A structural defect of idiopathic long OT syndrome. PACE 1991;14:1544-53.

14 Müller KD, Jakob $\mathrm{H}$, Neuzner J, et al. ${ }^{123} \mathrm{I}-$ metaiodobenzylguanidine scintigraphy in the detection of irregular regional sympathetic innervation in long QT syndrome. Eur Heart fै 1993;14:316-25.

15 Calkins $\mathrm{H}$, Lehmann $\mathrm{MH}$, Allman $\mathrm{K}$, et al. Scintigraphic pattern of regional cardiac sympathetic innervation in patients with familial long QT syndrome using positron emission tomography. Circulation 1993;87:1616-21.

16 Gill JS, Hunter GJ, Gane G, et al. Heterogeneity of the human myocardial sympathetic innervation: in vivo demby iodone-123-labeled meta-iodobenzylcintigraphy. Am Heart f 1993;126:390-8.

17 Tsuchimoto S, Tamaki N, Tadamura E, et al. Age and gender differences in normal myocardial adrenergic neuronal function evaluated by iodine-123-MIBG imaging. $7 \mathrm{Nucl}$ Med 1995;36:969-74.

18 Sisson JC, Shapiro B, Meyers L, et al. Metaiodobenzylguanidine to map scintigraphically the adrenergic nervous system in man. F Nucl Med 1987;28:1625-36.

19 Sanguinetti MC, Curran ME, Spector PS, et al. Spectrum of HERG $\mathrm{K}^{+}$-channel dysfunction in an inherited cardiac arrhythmia. Proc Natl Acad Sci USA 1996;93:2208-12.

20 Bennet PB, Yazawa K, Makita N, et al. Molecular mechanism for an inherited cardiac arrhythmia. Nature 1995;376:683-85.

21 De Ferrari GM, Nador F, Beria G, et al. Effects of calcium channel block on the wall motion abnormality of the idiopathic long QT syndrome. Circulation 1994;89:2126-32. 